\title{
Comment: Comparisons of reversal and nonreversal shifts'
}

PETER D. EIMAS

WILLIAMS COLLEGE

\begin{abstract}
Abstraet
It was argued that studies which employ comparisons between reversal (RV) and extradimensional(ED) shifts in order to test whether organisms use some mediational process during discriminative learning a re invalid for methodological reasons. However, adequate tests for mediation are possible from comparisons of intradimensional (ID) and ED shift problems. Data obtained from these comparisons support a mediational interpretation of discriminative learning.
\end{abstract}

In two recent articles (Tighe, Brown, \& Youngs, 1965; Tighe \& Tighe, 1965) the following results were obtained: (a) reversal (RV) shifts were more difficult than extradimensional (ED) shifts in rats with and without overtraining; and (b) without overtraining young children found the two transfer problems of approximately equal difficulty but with overtraining ED shifts were more difficult. They suggested that these data support a mediational theory of discriminative learning in which the mediational process is more readily available to organisms of higher phylogenetic position (cf. Kendler \& Kendler, 1962) and that overtraining "afforded the children additional opportunity to develop the appropriate mediating responses" (Tighe \& Tighe, 1965, p. 366).

The data are, however, confounded by a methodological error and consequently their conclusions must be re-examined. In both studies Ss were trained on Problem I with one dimension relevant, e.g., size, and one dimension irrelevant and variable, e.g., brightness. In Problem II the irrelevant dimension was made constani in both transfer conditions. The consequences of this change invalidate comparisons between the two shift conditions. Consider first the ED shift. If it were the case that in Problem I all Ss, in addition to acquiring appropriate instrumental responses, also learn some mediational response to the relevant but not to the irrelevant stimuli, then ED problems become markedly different probiems under constant as compared to variable irrelevant stimulation. For the dimension to which a mediational response has been learned, the relevant dimension of Problem I, is no longer differentiated in Problem II. Ss are forced to respond to the previously irrelevant dimension, now relevant, and the ED problem is no longer one in which Ss must first learn to extinguish one mediational response, learn another, and then learn the correct instrumental responses. Rather Ss need only learn a new mediating response plus appropriate instrumental responses. The ED problem is then easier under constant irrele- vant stimulation. The RV problem, on the other hand, has the same relevant dimension on Problems I and II; only the reinforcement values of the relevant stimuli are changed. Making the irrelevant dimension of Problem II constant does not, in all likelihood, change in any substantial manner the nature of the problem with respect to the mediating response. The finding of faster ED learning in all but one instance may be due to the fact the ED problem has been made easier and not that some Ss are unable to utilize mediating responses or do so only under certain conditions.

Moreover, resolution of the problem of the role of mediation in discriminative learning may very well not be possible by comparisons of RV and ED shifts. There may be no way to make the two conditions directly comparable as the following examples illustrate: (a) if variable irrelevant cues are used during transfer and these cues are the previously relevant cues of Problem I, then partial reinforcement effects will retard ED learning; (b) if two new cues are used to form the irrelevant dimension, then ED learning will again be interfered with due to the strong attraction of novel stimulation; and (c) if two new relevant and irrelevant cues are employed, then an unknown factor is introduced since it is operationally impossible to employ the same procedure for RV problems. The same relevant cues must necessarily be present in the RV problem.

There is a third transfer problem, intradimensional (ID) shift, which, when compared with the ED shift, permits an adequate test of whether an organism utilizes a mediational process during discriminative learning. An ID shift is defined as a transfer operation in which the relevant dimension of Problems I and II is the same as are the irrelevant dimensions. Only the particular relevant cue values must be changed in Problem II. However, if new relevant and irrelevant cues from the same dimensions are introduced in Problem II for ID and ED problems, then the sole differentiating factor between tasks is that of the relevant dimension, that is, whether it is the same or different from that of Problem I. Mediational theories, which postulate mediating responses, dimensional in nature, lead to predictions of faster acquisition of ID problems. Data from normal children (Eimas, 1965), retarded children (Campione, Hyman, \& Zeaman, 1963), and even rats (Shepp \& Eimas, 1964) have supported the proposition that all organisms mediate and their ability to do so is not related to either their phyloor ontogenetic status. 


\section{References}

Campione, J., Hyman, L., \& Zeaman, D. Dimensional shifts and reversals in retardate discrimination. In Learning and transfer in mental defectives. Progress Rep., No. 4, 1963, 134-142.

Eimas, P. D. Effects of overlearning, transfer, and age on the discrimination learning of children. Unpublished study, 1965.

Kendler, H. H., \& Kendler, T. S. Vertical and horizontal processes in problem solving. Psychol. Rev., 1962, 69, 1-16.

Shepp, B. E., \& Eimas, P. D. Intradimensional and extradimensional shifts in the rat. J. comp. physiol. Psychol., 1964, 57, 357361.

Tighe, L. S., \& Tighe, T. J. Overtraining and discrimination shift behavior in children. Psychon. Sci., 1965, 2, 365-366.

Tighe, T. J., Brown, P. L., \& Youngs, E. A. The effect of overtraining on the shift behavior of albino rats. Psychon. Sci., $1965,2,141-142$.

\section{Note}

1. Preparation of this paper was supported by Grant 1 RO1 HD00996-01 from the National Institute of Child Health and Human Development, United States Public Health Service.

\section{Reply to Eimas by T. J. Tighe and L. S. Tighe}

Eimas (1965) has apparently misread the purpose and the interpretation of our experiments (Tighe \& Tighe, 1965; Tighe, Brown, \& Youngs, 1965) and his comment ignores the chief question which is posed by the data obtained in these studies.

He has discussed the design of our experiments in relation to an hypothesis (i.e., that all Ss mediate) which was present neither in the purpose nor in the interpretation of the experiments in question. In fact (as clearly noted in the introductions to these experiments), the specific purpose of both studies was to determine the effect of additional training on the relative ease with which organisms accomplish those particular discrimination shifts (reversal: RS, and extradimensional: EDS) which have previously revealed species differences in performance under the specific procedure employed (e.g., compare Kelleher, 1956; Kendler \& Kendler, 1959; L. Tighe, 1965; T. Tighe, 1965). Thus there can be no question concerning the appropriateness of the designs used to the behavior studied and aims of these experiments.

In regard to his misunderstanding of an interpretation we advanced in our experiments, the following quotation from Eimas' comment is pertinent: "The finding of faster ED learning in all but one instance may be due to the fact that the ED problem has been made easier and not that some Ss are unable to utilize mediating responses or do so only under certain conditions" (italics ours). It should be noted that we did not make the italicized statements. Nothing in our interpretation of mediation theory held that some Ss are unable to mediate. Rather, we questioned the degree of development or availability of assumed mediating responses appropriate to the task in question, i.e., we questioned the sufficiency of mediation to enable more rapid RS than EDS under the conditions studied.

In commenting on the design and data of our experi- ments Eimas did not deal with the important point. He has detailed how an EDS with the irrelevant : nension constant (the procedure employed in our experiments) will be easier than an EDS with the irrelevant dimension varying, and has then gone on to suggest that the former condition may also produce faster EDS than RS. But he has dismissed completely our chief results in his statement that this procedural aspect may account for the finding in our experiments that EDS learning was faster than RS learning "in all but one instance" (italics ours). That is, Eimas did not deal with the finding that overtrained children exhibited faster RS than EDS while, to the contrary, EDS remained easier than RS in the case of overtrained rats. Furthermore Eimas was incorrect in stating that EDS was faster than RS in all but the one instance just discussed. In fact, EDS was not faster than RS in the case of children trained only to criterion; the difference in shift performance in this case did not approach significance $(p>.70)$. This result is important because it shows that in addition to the species difference in the effect of overtraining on shift performance there is also a difference in the shift performance of children and rats when both were trained only to criterion. In view of these species differences in relative ease of shift and in the effect of overtraining on relative ease of shift under the same testing procedures, it does not seem unreasonable to suggest the operation of some factor which varies with species. Nothing in Eimas' analysis questions the tenability of the hypothesis that this factor might be differences in the strengths or availability of appropriate mediating responses. And nothing in his analysis questions that such an hypothesis may be profitably investigated in this situation by such procedures as instating ari oiservable mediating response in organisms which typically exhibit relative ease of EDS, or by manipulating a variable which, according to the assumptions of some mediation theories, may be expected to affect the development of mediating processes (e.g., overtraining).

In the latter part of his comment Eimas also questioned the appropriateness of any form of RS-EDS comparison, advocating instead the use of a procedure which compares intradimensional shift (IDS) and EDS. The point and limits of this argument were not made clear, since the appropriateness of these designs is discussed in relation to the "... resolution of the problem of the role of mediation in discriminative learning." Our response to this section is that the two situations may be considered as providing different criteria of the existence and/or efficacy of an assumed mediating process. These criteria differ in the demands they place upon the assumed mediation process, but in the present state of knowledge both appear to be useful in the study of the role of mediation in discrimination learning. On the basis of the eminently reasonable assumption that there are differences as well as Continued on page 450. 